\title{
Casimir energy for de Sitter branes in bulk $\mathrm{AdS}_{5}$
}

\author{
Wade Naylor., M Misao Sasaki门 \\ Department of Earth and Space Science, Graduate School of Science, \\ Osaka University, Toyonaka 560-0043, Japan
}

\begin{abstract}
The vacuum energy for a massless conformally coupled scalar field in a brane world set up, corresponding to de Sitter branes in a bulk anti-de Sitter spacetime, is calculated. We use the Euclidean version of the metric which can be conformally related to a metric similar in form to the Einstein universe $\left(S^{4} \times R\right)$. Employing $\zeta$-function regularisation we evaluate the one-loop effective potential and show that the vacuum energy is zero for the one brane and non-zero for the two brane configuration. We comment on the back-reaction of this Casimir energy and on the inclusion of a mass term or non-conformal coupling.

PACS number(s): 04.50.+h, 11.25.Mj, 98.80.Cq
\end{abstract}

Many ideas and proposals within the circumference of what we call brane world cosmology (BWC) relate to the work of Randall and Sundrum (RS) [1] who initially implemented their two brane model to try and solve the hierarchy problem. In a variant of this model (a single brane with positive tension [2]) they also showed that the extra dimension could be of infinite extent. Generally speaking, the second model (and its extensions to include curved branes) has been of more interest to the cosmology community because gravity behaves essentially like 4-dimensional gravity localised near the brane [2, 迎.

An interesting BWC scenario has been developed in [5], where it is possible to obtain inflation on a single positive tension brane solely due to the effect of a bulk gravitational scalar field. In this regard, the vacuum energy of the bulk scalar field could have some affect on the cosmological evolution of the brane, (depending on the size and sign of this quantity) and is an interesting subject in its own right. The importance of vacuum energy in brane world scenarios (BWS) has also been highlighted in [6] where it has been suggested that it may be possible to solve the hierarchy problem with two positive tension branes, if the back-reaction of the Casimir effect is included (also see [7]).

In this letter we evaluate the one-loop effective potential (leading to the Casimir energy) for one and two de Sitter brane configurations in a bulk 5-dimensional anti-de Sitter spacetime. A massless conformally coupled scalar field is investigated as this is the simplest case technically. In [8] a similar calculation has been considered for spherical and hyperbolic one brane configurations. However, we consider a spherical two brane configuration and for the one brane case the results agree. Quite extensive work has been done for flat branes and we refer the reader to the literature, see [9] 14] and the references therein.

Calculations of the Casimir energy are non-intuitive in how the overall sign depends on the topology and/or dimension of the spacetime [15. One has to perform the calculation. To obtain the result, we employ the $\zeta$-function regularisation method [16. The beauty of $\zeta$-function regularisation (or some would say its weakness) is that it automatically removes the divergences of the theory leaving a finite result. An interesting discussion about different analytic continuations in $\zeta$-function regularisation can be found in [17].

To perform our calculation we use the Euclideanised form of the metric suitable for de Sitter branes [18]. It can be written in conformal form as

$$
d s^{2}=a^{2}(z)\left(d z^{2}+d \chi^{2}+\sin ^{2} \chi d \Omega_{(3)}^{2}\right) ; \quad a(z)=\frac{\ell}{\sinh \left(z_{0}+|z|\right)},
$$

where $\ell=\left(-6 / \Lambda_{5}\right)^{1 / 2}$ is the anti-de Sitter radius, $d \Omega_{(3)}^{2}$ is the metric on the 3 -sphere and the positive tension brane is placed at $z=0$. Here, we place the other negative tension brane at $|z|=L$ (the one brane configuration is given by $L \rightarrow \infty$ ). The brane tensions in this case are 18,19.

\footnotetext{
${ }^{*}$ E-mail:wnaylor@vega.ess.sci.osaka-u.ac.jp

${ }^{\dagger}$ E-mail:misao@vega.ess.sci.osaka-u.ac.jp
} 


$$
\sigma_{+}=\frac{3}{4 \pi G_{5} \ell} \cosh z_{0}, \quad \sigma_{-}=-\frac{3}{4 \pi G_{5} \ell} \cosh \left(z_{0}+L\right),
$$

and the non-dimensional length $L$ is given in terms of the physical length $r$ as

$$
L=\int_{r_{-}}^{r_{+}} \frac{d r}{a}=\int_{r_{-}}^{r_{+}} \frac{d r}{\ell \sinh (r / \ell)} .
$$

The metric is conformal to the Euclidean version of an Einstein universe $\left(S^{4} \times R\right)$ with unit radius. The topology of this metric corresponds to two concentric hyperspheres of equal radius on a cylinder of length $L$ (the conformal scaling has shrunk the radii of the two hyperspheres to equal radius).

Because we are dealing with a conformally coupled scalar field we must

find the eigenvalues of the Klein-Gordon equation given by (e.g., see [20])

$$
\begin{aligned}
\left(-\partial_{z}{ }^{2}-\Delta^{(4)}+\xi_{5} R^{(4)}\right) \phi & =\lambda^{2} \phi, \\
{\left[\left(\frac{\pi n}{L}\right)^{2}-\Delta^{(4)}+\frac{9}{4}\right] \phi } & =\lambda^{2} \phi,
\end{aligned}
$$

where $\xi_{5}=3 / 16, R^{(4)}=12$ and imposing $Z_{2}$ symmetry on the branes implies Neumann boundary conditions giving the discrete sum between the brane boundaries, for a conformally coupled scalar field [10]. In general, as pointed out in [8], the eigenfunctions will satisfy mixed boundary conditions and the spectrum of $-\bar{\partial}_{z}^{2}$ will not be easily obtained. For the operator $-\Delta^{(4)}+9 / 4$, the eigenvalues are (for Gegenbauer polynomials see [21].)

$$
\lambda_{m}^{2}=(m+3 / 2)^{2}, \quad d(m)=\frac{1}{3}(m+2)(m+3 / 2)(m+1),
$$

where $d(m)$ is the degeneracy. Thus the eigenvalues for the Klein-Gordon operator are

$$
\lambda_{n, m}^{2}=\left(\frac{\pi n}{L}\right)^{2}+(m+3 / 2)^{2} .
$$

Employing the Euclidean effective action, we define the function

$$
g(s)=\sum_{m, n=0}^{\infty} d(m) \lambda_{m, n}^{-2 s} .
$$

and then the one-loop effective action, $W_{1}$, is related to $g(s)$ by

$$
W_{1}=-\frac{1}{2} g^{\prime}(0) \text {. }
$$

Note, there is no renormalisation scale, $\mu$, in the function $g(s)$ because the eigenvalues are dimensionless (due to the conformal rescaling).

We first begin with the simpler case of the vacuum energy for the Einstein universe $S^{4} \times R$. This is equivalent to finding the zero point energy of the equation

$$
\partial_{t}^{2} \psi-\Delta^{(4)} \psi+\frac{3}{16} R^{(4)} \psi=0
$$

The eigenvalues and degeneracy are still given by Eq. (E). It is then straightforward to show that

$$
E_{0}=\frac{1}{2} g(-1 / 2)=\frac{1}{6}\left(\zeta(2 s-3,3 / 2)-\frac{1}{4} \zeta(2 s-1,3 / 2)\right)_{s \rightarrow-1 / 2}=0
$$

where $\zeta(a, b)$ is the generalised (or Hurwitz) $\zeta$-function and is related to the Bernoulli polynomial $B_{n}(x)$ by [21]

$$
\zeta(-n, a)=-\frac{B_{n+1}(a)}{n+1} .
$$

It is easy to verify that $E_{0}$ is zero, using Eq. (11), as should be expected because in an odd number of dimensions there is no conformal anomaly [20]. This calculation is similar to the one by Ford [22] for $S^{3} \times R$, where an exponential cut off was used in the mode sum rather than $\zeta$-function regularisation (for $S^{2} \times R$ see [23]).

We now evaluate the effective potential for a one brane configuration, where $L \rightarrow \infty$ in Eq. (何) and the discrete sum over $n$ is now an integral. The function $g(s)$ becomes 


$$
g_{1}(s)=\frac{2 L}{\pi} \int_{0}^{\infty} d k \sum_{m=0}^{\infty} d(m)\left(k^{2}+(m+3 / 2)^{2}\right)^{-s}
$$

where the factor of 2 is because there are two copies of the bulk space on either side of the brane. For large $s$ we can interchange the order of the sum and the integral and perform the $k$ integration, leading to

$$
\begin{aligned}
g_{1}(s) & =\frac{2 L}{\pi} \frac{\sqrt{\pi}}{2} \sum_{m=0}^{\infty} \frac{\Gamma(s-1 / 2)}{\Gamma(s)} d(m)(m+3 / 2)^{1-2 s}, \\
& =\frac{L}{\pi} \frac{\sqrt{\pi}}{3} \frac{\Gamma(s-1 / 2)}{\Gamma(s)}\left(\zeta(2 s-4,3 / 2)-\frac{1}{4} \zeta(2 s-2,3 / 2)\right),
\end{aligned}
$$

where in the second step we have used simple algebra to rewrite the equation in terms of generalised $\zeta$-functions. For $s=0$ it is clear that $g_{1}(0)=0$ because

$$
\frac{1}{\Gamma(s)}=s+\gamma s^{2}+O\left(s^{3}\right)
$$

where $\gamma$ is Euler's constant. Thus,

$$
g_{1}^{\prime}(0)=\frac{L}{\pi} \frac{\sqrt{\pi}}{3} \Gamma(-1 / 2)\left(\zeta(-4,3 / 2)-\frac{1}{4} \zeta(-2,3 / 2)\right)=0,
$$

which is zero because the combination of $\zeta$-functions in Eq. (15) cancel, as can be verified by employing Eq. (11). Therefore, the one-loop effective potential (and hence the vacuum energy) for a conformally coupled scalar field is zero for the one brane case and does not affect BWC models, such as in [5]. This is consistent with the result found in [8]. Although, one might naively expect imposing a boundary on the brane similar to that for a half Einstein universe will give a non-zero contribution.

Now we come to the main task at hand. The two brane configuration has two infinite summations, which can be expressed in terms of generalised $\zeta$-functions. In fact, there are many subtle issues regarding the correct analytic continuation of such functions upon interchange of the order of the summations (see [16] for a detailed discussion). The function $g(s)$ becomes

$$
\begin{aligned}
g_{2}(s) & =\sum_{n=-\infty}^{\infty} \sum_{m=0}^{\infty} d(m)\left(c^{2} n^{2}+(m+3 / 2)^{2}\right)^{-s} \\
& =\frac{1}{\Gamma(s)} \sum_{n=-\infty}^{\infty} \sum_{m=0}^{\infty} d(m) \int_{0}^{\infty} d t t^{s-1} \exp \left\{-t\left[c^{2} n^{2}+(m+3 / 2)^{2}\right]\right\},
\end{aligned}
$$

where $c=\pi / L$ and in the second step we have made use of the Mellin transform. The sum over $n$ is from $-\infty$ to $\infty$ because imposing orbifold boundary conditions essentially doubles the modes. This formula is almost identical in form to the 2-dimensional Epstein-Hurwitz $\zeta$-function studied by Elizalde in [24] (see also [16]), apart from the degeneracy factor $d(m)$. This work has many interesting results and the one we employ is

$$
\sum_{n=-\infty}^{\infty} e^{-c^{2} n^{2}}=\sqrt{\frac{\pi}{c^{2}}}+2 \sqrt{\frac{\pi}{c^{2}}} \sum_{n=1}^{\infty} \exp \left[-\pi^{2} n^{2} / c^{2}\right]
$$

This is non other than the unimodular transformation formula of Jacobi's $\theta_{3}$ function [21] (the Poisson resummation formula). Substitution of the above equation into (16) allows us to interchange the order of the summations. After integrating with respect to $t$ and performing some simple algebra we obtain

$$
\begin{aligned}
g_{2}(s)= & +\frac{1}{3} \sqrt{\frac{\pi}{c^{2}}} \frac{\Gamma(s-1 / 2)}{\Gamma(s)}\left(\zeta(2 s-4,3 / 2)-\frac{1}{4} \zeta(2 s-2,3 / 2)\right) \\
& +\frac{4 \pi^{s}}{\Gamma(s)} c^{-s-1 / 2} \sum_{n=1, m=0}^{\infty} d(m) n^{s-1 / 2}(m+3 / 2)^{-s+1 / 2} K_{s-1 / 2}\left[2 \pi c^{-1} n(m+3 / 2)\right] .
\end{aligned}
$$

It is easy to verify that $g_{2}(0)=0$, using Eq. (14). Furthermore, taking the derivative of $g_{2}(s)$ with respect to $s$ (and leaving only terms that remain independent of $\Gamma(s)$ near $s=0$, see Eq. (14)) gives

$$
\begin{aligned}
g_{2}^{\prime}(0)= & +\frac{1}{3} \sqrt{\frac{\pi}{c^{2}}} \Gamma(-1 / 2)\left(\zeta(-4,3 / 2)-\frac{1}{4} \zeta(-2,3 / 2)\right) \\
& +4 c^{-1 / 2} \sum_{n=1, m=0}^{\infty} d(m) n^{-1 / 2}(m+3 / 2)^{1 / 2} K_{-1 / 2}\left[2 \pi c^{-1} n(m+3 / 2)\right]
\end{aligned}
$$


where a prime denotes differentiation with respect to $s$. Interestingly, the first term is exactly the contribution from the one brane configuration Eq. (15), which is zero. This is similar to finite temperature field theory where the $n=0$ mode gives the zero temperature contribution (this can be clearly seen by using heat kernel methods [25], see also [8]). Of course, this kind of behaviour was expected due to the topology of the conformally re-scaled metric, Eq. (1). The second term is non-zero and depends on the value of $c=\pi / L$. Note that $K_{-1 / 2}(z)=\sqrt{\pi /(2 z)} e^{-z}$. Therefore, the one-loop effective potential per unit volume is given by, after some simplification,

$$
V_{1}=\frac{W_{1}}{2 L \operatorname{Vol}\left(\mathrm{S}^{4}\right)}=-\frac{A(L)}{8 \pi^{2} L}
$$

where $W_{1}$ is the effective action for the conformally re-scaled field and

$$
\begin{aligned}
A(L) & =\sum_{n=1, m=0}^{\infty} \frac{(m+2)(m+3 / 2)(m+1)}{n} \exp [-2 \operatorname{Ln}(m+3 / 2)] \\
& =-\sum_{m=0}^{\infty}(m+2)(m+3 / 2)(m+1) \ln (1-\exp [-L(2 m+3)]) .
\end{aligned}
$$

The function $A(L)$ is generally a complicated function of $L$, but its qualitative behaviour can be deduced analytically. First, it is easy to see that $A(L)>0$ and $d A(L) / d L<0$ for $L>0$. Note, that we have normalized the radius of $\mathrm{S}^{4}$ to unity. Therefore, the limit $L \rightarrow 0$ corresponds to infinitely large 4 -spheres on the boundaries, i.e., flat space. In the limit $L \ll 1$, we may approximate $A(L)$ as

$$
\begin{aligned}
A(L) & \approx-\int_{0}^{\infty} d m m^{3} \ln (1-\exp [-2 m L]) \\
& =\frac{1}{4(2 L)^{4}} \int_{0}^{\infty} d x \frac{x^{4}}{e^{x}-1}=\frac{\Gamma(5) \zeta(5)}{4(2 L)^{4}},
\end{aligned}
$$

which gives

$$
V_{1} \approx-\frac{\Gamma(5) \zeta(5)}{2^{10} \pi^{2} L^{5}} \quad \text { for } \quad L \ll 1
$$

Using the relation,

$$
\zeta^{\prime}(-2 n)=(-1)^{n} \frac{\Gamma(2 n+1) \zeta(2 n+1)}{2(2 \pi)^{2 n}} \quad(n=1,2,3, \cdots),
$$

we find Eq. (23) exactly agrees with the flat space Casimir energy for a conformal scalar [10]. It may be worth mentioning that $V_{1}$ given by Eq. (23) is also equal to the free energy of a massless boson at temperature $T=1 /(2 L)$, except for the factor of 2 that comes from the mode doubling. In the limit $L \gg 1, A(L)$ behaves as

$$
A(L) \approx \sum_{m=0}^{\infty}(m+2)(m+3 / 2)(m+1) \exp [-L(2 m+3)] \sim 3 \exp [-3 L] .
$$

Thus, the Casimir energy for de Sitter boundaries decreases much more rapidly than that for flat space boundaries as the non-dimensional length $L$ increases. We have checked our results for the sum $A(L)$ on different numerical packages and find that, for example, when $L=0.5,1$, and $2, A(L)=5.90,0.31$ and 0.008 , respectively.

From the symmetries of the metric, Eq. (1), and because the stress energy tensor for a conformally coupled scalar field is traceless in odd dimensions (no conformal anomaly, see [20]) the Casimir energy density, $\rho$, is equal to the effective potential, $V_{1}$. Also, using the fact that the conformally re-scaled stress energy tensor is given in terms of the original one by $\left\langle T^{\mu}{ }_{\nu}\right\rangle_{g}=a^{-D}\left\langle T^{\mu}{ }_{\nu}\right\rangle$, see [20,10], we have

$$
\left\langle T^{\mu}{ }_{\nu}\right\rangle_{g}=\ell^{-5} \sinh ^{5}\left(z_{0}+|z|\right)\left\langle T^{\mu}{ }_{\nu}\right\rangle .
$$

This then implies that the physical energy density $\rho_{g}$ is given by

$$
\rho_{g}=-\frac{\sinh ^{5}\left(z_{0}+|z|\right)}{8 \pi^{2} \ell^{5} L} A(L) .
$$

We now discuss the implications of these analyses on the issues raised in the introduction.

The back-reaction of the Casimir energy plays an important role in BWS [6.7], including inflating branes. However, in [6] the back-reaction for the de Sitter brane model only considered the vacuum energy from Minkowski branes. For 
flat branes this only depends on the difference between the number of bosons $\left(N_{b}\right)$ and fermions $\left(N_{f}\right)$, where $N_{b}=N_{f}$ gives the usual RS model and $N_{b}>N_{f}$ gives two positive tension branes, see [6]. Thus, we should also consider other fields such as the fermion. However, in contrast to Minkowski branes, the eigenvalues in the mode sum for two de Sitter branes are different for bosons and fermions, see 22] for $S^{3} \times R$, implying the vacuum energy will not be of equal magnitude, but opposite in sign. Therefore, we can infer, from the previous sentences, that the bulk will not remain pure anti-de Sitter under the back-reaction (for de Sitter branes) even for $N_{b}=N_{f}$.

In the context of BWC models, such as in [5], we must consider the more general case of a massive or non-conformal scalar field. Adding a mass term will break conformal invariance. Altering the conformal coupling constant $\xi_{5}$ not only breaks conformal invariance, but also modifies the boundary conditions. As we mentioned earlier (also see [8]), the boundary conditions will become mixed. One can of course consider a small perturbation of $\xi_{5}$ or a small mass term. Such cases may be treated analytically. Otherwise, the eigenspectrum of $-\partial_{z}^{2}$ needs to be solved numerically. Nevertheless, for large $n$ the spectrum will return to $\pi n / L$ and we expect to need only evaluate this correction to the Casimir energy up to, say, $n \approx 10$.

In forthcoming work [25] we will present a more detailed discussion of the $\zeta$-function regularisation procedure. There, its relation to the heat kernel will be made explicit. In connection with the generalisation to non-conformal cases, we mention the work done by Dowker and Apps [26] for generalised cylinders (up to 4-dimensions), and the work by Garriga, Pujolàs and Tanaka [10,27] for a 5-dimensional spacetime bounded by flat branes. They stress the importance of the cocycle function. Also, we will present the results for a spinor field and an analysis of the introduction of a small mass term (or non-conformal coupling) into the wave equation. Other avenues of research for de Sitter branes include the general non-conformal case, finite temperature effects (see [28] for the RS model and also the comments made in [29]), boundaries on the brane, and the possibility of stabilising the radion field.

\section{ACKNOWLEDGMENTS}

W.N. acknowledges support from JSPS for Postdoctoral Fellowship for Foreign Researchers No. P01773. The work of M.S. is supported by Monbukagaku-sho Grant-in-Aid for Scientific Research (S) No. 14102004. We are grateful to Ian Moss for useful discussions and drawing our attention to reference [26]. We would also like to thank Yoshiaki Himemoto, Alan Knapman and Takahiro Tanaka for interesting conversations.

[1] L. Randall, R. Sundrum, Phys. Rev. Lett. 83 (1999) 3370.

[2] L. Randall, R. Sundrum, Phys. Rev. Lett. 83 (1999) 4690.

[3] T. Shiromizu, K. I. Maeda, M. Sasaki, Phys. Rev. D 62 (2000) 024012.

[4] J. Garriga, T. Tanaka, Phys. Rev. Lett. 84 (2000) 2778.

[5] Y. Himemoto, M. Sasaki, Phys. Rev. D 63 (2001) 044015.

[6] S. Mukohyama, Phys. Rev. D 63 (2001) 044008.

[7] R. Hofmann, P. Kanti, M. Pospelov, Phys. Rev. D 63 (2001) 124020.

[8] S. Nojiri, S. D. Odintsov, S. Zerbini, Class. Quant. Grav. 17 (2000) 4855.

[9] D. J. Toms, Phys. Lett. B 484 (2000) 149.

[10] J. Garriga, O. Pujolàs, T. Tanaka, Nucl. Phys. B 605 (2001) 192.

[11] W. D. Goldberger, I. Z. Rothstein, Phys. Lett. B 491 (2000) 339.

[12] A. Flachi, D. J. Toms Nucl. Phys. B 610 (2001) 144.

[13] A. Flachi, I. G. Moss, D. J. Toms, Phys. Lett. B 518 (2001) 153.

[14] A. Flachi, I. G. Moss, D. J. Toms, Phys. Rev. D 64 (2001) 105029.

[15] K. A. Milton, "The Casimir effect: Physical manifestations of zero-point energy," River Edge, USA: World Scientific (2001).

[16] E. Elizalde, "Ten physical applications of spectral zeta functions," Lect. Notes Phys. M35 (1995).

[17] B. F .Svaiter, N. F .Svaiter, Phys. Rev. D 47 (1993) 4581.

[18] J. Garriga, M. Sasaki, Phys. Rev. D 62 (2000) 043523.

[19] U. Gen, M. Sasaki, Prog. Theor. Phys. 105 (2001) 59.

[20] N. D. Birrel, P. C. .W. Davies, "Quantum fields in curved space," Cambridge University Press (1981).

[21] A. Erdélyi ed., "Higher transcendental functions," McGraw-Hill, reprint edition (1981).

[22] L. H. Ford, Phys. Rev. D 11 (1975) 3370.

[23] S. S. Bayin, M. Özcan, J. Math. Phys. 38 (1997) 5240.

[24] E. Elizalde, J. Math. Phys. 31 (1990) 170.

[25] I. G. Moss, W. Naylor, W. Santiago-Germàn, M. Sasaki, paper in preparation.

[26] J. S. Dowker, J. S. Apps, Class. Quant. Grav. 12 (1995) 1363.

[27] J. Garriga, O. Pujolàs, T. Tanaka, arXiv:hep-th/0111277. 
[28] I. Brevik, K. A. Milton, S. Nojiri, S. D. Odintsov, Nucl. Phys. B 599 (2001) 305.

[29] I. Z. Rothstein, Phys. Rev. D 64 (2001) 084024. 